* Corresponding author E-mail address: ladislav.starek@stuba.sk (Ladislav Starek, prof., Ing., PhD.)

Article information Article history: AMS-Volume15-No.3-00116-11 Received 21 May 2011 Accepted 18 June 2011

\section{Application of Inverse Vibration Problems to Model Updating}

\author{
Ladislav Starek ${ }^{\mathrm{a} *}$, D.J. Inman ${ }^{\mathrm{b}}$, Daniel Starek ${ }^{\mathrm{c}}$ \\ a Institute of Applied Mechanics and Mechatronic, Faculty of Mechanical Engineering, Slovak University of Technology at Bratislava, 81231 \\ Bratislava, Slovakia \\ ${ }^{b}$ Center for Intelligent Material Systems and Structures Department of Mechanical Engineering, Virginia Polytechnic Institute and State \\ University Blacksburg, VA 24061 \\ a Department of Structural Mechanics, Faculty of Civill Engineering, Slovak University of Technology at Bratislava, 81231 Bratislava, Slovakia
}

\section{BIOGRAPHICAL NOTES}

Prof. Ing. Ladislav Starek, PhD., born in 1950, was graduated with M.Sc. in Mechanical Engineering from Slovak Technical University in 1973. In the same year he started work on a Ph.D. in Mechanics of rigid and elastic bodies at Slovak Technical University which was awarded in 1978. He then worked for the next three years as a Senior lecturer in subjects of Mechanics of rigid bodies at the Department of Technical Mechanics, Faculty of Mechanical Engineering, Slovak Technical University. In the fall of 1981 he was promoted to associate professor at the same faculty. He has been teaching and performing research in the area of Mechanics of rigid and elastic bodies and Vibration for undergraduate and postgraduate students. In 1997 he was appointed Professor in Applied Mechanics. He was as supervisor for nine postgraduate students. Prof. Starek is a professor at Slovak University of Technology Bratislava and has authored over 100 journal and proceedings articles since receiving his Ph.D. in 1978. He teaches and performs research in the areas of vibration of mechanical systems in particular vibration with control, suppression of vibration, inverse problem in vibration. Starek has authored 8 temporary texts which were introduced by Slovak Technical University. In 2009 was published his book Vibration with Control. The most important publication outputs include articles published in journals ASME Journal of Applied Mechanics, Journal of Sound and Vibration, and others. During 1994-2007 he was acting as head of Department of Technical Mechanics of Faculty of Mechanical Engineering, Slovak University of Technology at Bratislava. Now he is working as a professor of Institute Applied Mechanics and Mechatronics.

Ing. Daniel Starek, PhD., born in 1982, was graduated with M.Sc. in Faculty of Mechanical Engineering, Slovak Technical University at Bratislava in 2005. In the same year he started work on a Ph.D. in Mechatronics at Slovak Technical University, Faculty of Mechanical Engineering, Department of Technical Mechanics. The subject of his research is application of SMART materials (especially piezoceramics and rheological fluids) to suppression of vibration of mechanical structures. D. Starek is a reseach worker at Slovak University of Technology at Bratislava and has authored 7 journal and proceedings articles since entering Department of Technical Mechanics in 2005. He carrying out research in the area of suppression of vibration of mechanical systems. Since 2010 D. Starek is with Department of Structures, Faculty of Civil Engineering, Slovak Univerzity of Technology. D. Starek is leading seminars from subjects of Applied Mechanics at undergraduate course at the Faculty of Civil Engineering. 


\section{KEY WORDS}

Vibration, Eigenvalue problem, Inverse eigenvalue problem

\section{ABSTRACT}

This paper summarize the authors previous effort on inverse eigenvalue problem and applies the theory to the model updating problem. Comments are made on how their procedure may be used to solve the damage detection problem.

\section{Introduction}

Here we consider linear lumped parameter system which can be modeled by a vector differential equation in the second order form given by

$$
M \ddot{q}(t)+D \dot{q}(t)+K q(t)=f_{1}(t)
$$

where $\boldsymbol{q}(\boldsymbol{t})$ is an $\boldsymbol{n}$ vector of time-varying elements representing the displacement of the masses in a lumped mass model of some structure or device. The vectors $\dot{\boldsymbol{q}}(\boldsymbol{t})$ and $\ddot{\boldsymbol{q}}(\boldsymbol{t})$ represent the velocities and accelerations, respectively. The $\boldsymbol{n}$ vector $\boldsymbol{f}_{\boldsymbol{l}}(\boldsymbol{t})$ represents applied external forces and its time varying. The coefficients $\boldsymbol{M}, \boldsymbol{D}$ and $\boldsymbol{K}$ are $\boldsymbol{n} \boldsymbol{x} \boldsymbol{n}$ matrices of constant real elements representing the various physical parameters of mass, damping, and stiffness. The matrices $\boldsymbol{M}, \boldsymbol{D}$ and $\boldsymbol{K}$ could be either asymmetric or symmetric and if symmetric $\boldsymbol{M}$ is positive definite.

If $\boldsymbol{M}$ is positive definite and symmetric it has a positive definite matrix square root, with a symmetric, positive definite inverse denoted by $\boldsymbol{M}^{-1 / 2}$. Let us then consider the transformation $q(t)=M^{-1 / 2} \nu(t)$. Substitution of this change of coordinates into Eq. (1) yields

$$
\ddot{v}(t)+\tilde{D} \dot{v}(t)+\tilde{K} v(t)=p(t)
$$

where $\tilde{K}=M^{-1 / 2} K M^{-1 / 2}$ and $\tilde{D}=M^{-1 / 2} D M^{-1 / 2}$ are necessarily symmetric if $\mathbf{D}$ and $\mathbf{K}$ are and $p(t)=M^{-1 / 2} f_{1}(t)$.

Previously, inverse spectral problems in vibration of lumped nonconservative systems have been solved in [1], [2], [4], [5], [7], [8], [9], [11].

In [4] the inverse spectral problem for vibration of lumped conservative systems $(\tilde{D}=0)$ modeled by tridiagonal matrices has been solved. In [1] has been solved this problem for the case of real nonsingular coefficient matrices and he has defined the inverse formulas which determine the coefficient matrices $\boldsymbol{M}, \boldsymbol{D}$ and $\boldsymbol{K}$ of the above mentioned systems with given spectral and modal data. In [7] has been solved the inverse problem in the state space form and authors have determined the inverse formulas which directly determine real coefficient matrices $\boldsymbol{M}^{-1} \boldsymbol{K}$ and $\boldsymbol{M}^{-1} \boldsymbol{D}$ for the case that $\boldsymbol{D}$ and $\boldsymbol{K}$ are singular coefficient matrices (i.e., there exist rigid-body modes). The symmetric inverse problem with overdamped modes has been discussed in [9].

The results presented here build on those of Lancaster and Maroulas [5], those of Starek and Inman [8] and of those of Starek and Inman [1 1]. Lancaster and Maroulas have solved the inverse problem in vibration by means of the spectral theory of matrix polynomials. They defined Jordan pairs that determine a self-adjoint matrix polynomial. Starek and Inman [8] have been solved the inverse spectral problems in the state space form and defined the conditions for given spectral and modal data under which the inverse formulas determine real symmetric coefficient matrices $\tilde{D}$ and $\tilde{K}$. However, their solution requires that the given eigenvalues must all be complex valued and does not preserve given eigenvectors.

The paper Starek and Inman [11] gives an alternative solution to the inverse problem solved in Starek and Inman [8] and extends these results to include the preservation of eigenvectors. They have derived conditions under which spectral and modal data determine real, symmetric coefficient matrices $\tilde{D}$ and $\tilde{K}$ which do not necessarily commute. The method has been outlined which allows the synthesis of a symmetric, positive semidefinite underdamped linear system having desired eigenvalues and eigenvectors [2], [10]. The goal of this paper is to give an alternative solution to the inverse eigenvalue problem in vibration in $\mathbf{2} \boldsymbol{n}$ space to include the determination of all three coefficient matrices $\boldsymbol{M}, \boldsymbol{D}$ and $\boldsymbol{K}$ from given spectral and modal data and to apply the inverse eigenvalue problem to the model updating problem [9], [11].

\subsection{Symmetric Case}

\section{Theoretical Background}

By combining the vector identity $\dot{v}(t)-\dot{v}(t)=0$ with Eq. (2), this system can be written in first order $2 \boldsymbol{n}$ space as

$$
N \dot{u}(t)-P u(t)=g(t)
$$


where the vector $\boldsymbol{u}(\boldsymbol{t})$ and $\boldsymbol{g}(\boldsymbol{t})$ are defined by

$u(t)=\left[\begin{array}{c}v(t) \\ v(t)\end{array}\right]$, and $g(t)=\left[\begin{array}{c}p(t) \\ 0\end{array}\right]$

and the coefficient matrices are defined by the partitioned forms

$N=\left[\begin{array}{cc}\tilde{D} & I \\ I & 0\end{array}\right]$, and $P=\left[\begin{array}{cc}-\tilde{K} & 0 \\ 0 & I\end{array}\right]$

Finally, pre-multiply Eq. (3) by the inverse matrix $N^{-1}$. This yields the standard state space formulation

$\dot{x}(t)=A x(t)+h(t)$

where the state vector $\boldsymbol{x}(\boldsymbol{t})=\boldsymbol{u}(\boldsymbol{t}), \boldsymbol{h}(\boldsymbol{t})=\boldsymbol{N}^{-1} \boldsymbol{g}(\boldsymbol{t})$ and the state matrix is given by

$A=\left[\begin{array}{cc}0 & I \\ -\tilde{K} & -\tilde{D}\end{array}\right]$

Note that the inverse of the matrix $\boldsymbol{N}$ is given by the partitioned form

$N^{-1}=\left[\begin{array}{cc}0 & I \\ I & -\tilde{D}\end{array}\right]$

and hence exists whether or not the matrix $\tilde{D}$ is singular.

The three eigenvalue problems are

$$
\begin{aligned}
& \left(\tilde{K}+\lambda \tilde{D}+\lambda^{2} I\right) v=0 \\
& (P-\lambda N) u=0 \\
& (A-\lambda I) x=0
\end{aligned}
$$

where the constant vectors $\boldsymbol{v}, \boldsymbol{u}$ and $\boldsymbol{x}$ must be nonzero. Equation (8) defines the second order matrix polynomial problem, Eq. (9) defines the (first order) matrix pencil problem and Eq. (10) defines the standard matrix eigenvalue problem. Each of these three problems results in the same set of $\mathbf{2 n}$ complex scalars, denoted $\lambda_{\mathbf{i}^{\prime}}$ which are the eigenvalues of the system, and which contain the system's natural frequencies and damping ratios. Each of the complex vectors $\boldsymbol{v}_{\mathbf{i}^{\prime}} \boldsymbol{u}_{\mathbf{i}}$ and $\boldsymbol{x}_{\mathbf{i}}$ corresponding to each $\lambda_{\mathrm{i}}$ are in some way related to the system's mode shapes.

Each of these eigenvalue problems in $\boldsymbol{v}_{\mathbf{i}^{\prime}} \boldsymbol{u}_{\mathbf{i}}$ and $\boldsymbol{x}_{\mathbf{i}}$ can be restated as a matrix equation by defining the matrix $\Lambda$ to be the $\mathbf{2} \boldsymbol{n} \times \mathbf{2} \boldsymbol{n}$ diagonal matrix of eigenvalues $\lambda_{\mathrm{i}}$ with 1's on the super diagonal if a particular $\lambda_{\mathrm{i}}$ is not simple. In addition, if the $\boldsymbol{n} \times \mathbf{2} \boldsymbol{n}$ matrix $\boldsymbol{V}$ is defined by taking the $\mathbf{2} \boldsymbol{n}, \boldsymbol{n} \times \mathbf{1}$ vectors $\mathbf{v}_{\mathbf{i}}$ as its columns, if the $\mathbf{2} \boldsymbol{n} \times \mathbf{2} \boldsymbol{n}$ matrix $\boldsymbol{U}$ is defined by taking the $\mathbf{2} \boldsymbol{n}, \mathbf{2} \boldsymbol{n} \times \mathbf{1}$ vectors as its columns, and if the $2 \boldsymbol{n} \times \mathbf{2} \boldsymbol{n}$ matrix $\boldsymbol{X}$ is defined by taking the $2 \boldsymbol{n}, 2 \boldsymbol{n} \times \mathbf{1}$, vectors $\mathbf{x}_{\mathbf{i}}$ as its columns, then Eqs. (8), (9), and (10) can be expressed as the matrix equations:

$$
\begin{aligned}
& \tilde{K} V+\tilde{D} V \Lambda+V \Lambda^{2}=0 \\
& P U-N U \Lambda=0 \\
& A X-X \Lambda=0
\end{aligned}
$$

\subsection{Asymmetric Case and Inverse Formulas for all Three Coefficient Matrices}

Bycombining thevectoridentity $M \dot{q}(t)-M \dot{q}(t)=0$ with equation (1), this system can be written in the first order $\mathbf{2} \boldsymbol{n}$ space as is given by (3) where

$\mathrm{u}(\mathrm{t})=\left[\begin{array}{l}q(t) \\ \dot{q}(t)\end{array}\right], \mathrm{g}(t)=\left[\begin{array}{l}\mathrm{f}(t) \\ 0\end{array}\right]$

and the matrices

$\boldsymbol{P}$ and $\boldsymbol{N}$ are defined by the partitioned form

$\bar{P}=\left[\begin{array}{cc}-\mathrm{K} & 0 \\ 0 & \mathrm{M}\end{array}\right], \bar{N}=\left[\begin{array}{cc}\mathrm{D} & \mathrm{M} \\ \mathrm{M} & 0\end{array}\right]$

Finally, pre-multiply Eq. (3) by the inverse ma$\operatorname{trix} \boldsymbol{N}^{-1}$. This yields the standard state space formulation given by (5) where the state vector $x(t)=u(t), h(t)=N^{-1} g(t)$ and the state matrix $A$ is given by

$A=\left[\begin{array}{cc}0 & \mathrm{I} \\ -\mathrm{M}^{-1} \mathrm{~K} & -\mathrm{M}^{-1} \mathrm{D}\end{array}\right]$

Eigenvalue problems for that case can be expressed as the matrix equation

$A X-X \Lambda=0=Y^{T} A-\Lambda Y^{T}$

$P U_{r}-N U_{r} \Lambda=0=U_{l}^{T} P-\Lambda U_{l}^{T} N$

where the matrices $\boldsymbol{U}_{1}$ and $\boldsymbol{Y}$ are the matrices of left eigenvectors of the systems given by Eqs. (3) and (5). The orthogonality and norm conditions are defined as follows

$Y^{T} A X=\Lambda, Y^{T} X=I$ 
$U_{l}^{T} P U_{r}=\Lambda, U_{l}^{T} N U_{r}=I$

$W^{T} D V+W^{T} M V \Lambda+\Lambda W^{T} M V=I$

From the Eq. (14), (15), (19) and (20) follows:

$X=U_{r}, U_{r}=\left[\begin{array}{c}V \\ V \Lambda\end{array}\right], U_{l}=\left[\begin{array}{c}W \\ W \Lambda^{T}\end{array}\right]$

$Y^{T}=U_{l}^{T} N$

$A=X \Lambda Y^{T}=U_{r} \Lambda U_{l}^{T} N$

After some manipulation we get

$M=\left(V \Lambda W^{T}\right)^{-1}$

$D=-M\left(V \Lambda^{2} W^{T}\right) M$

$K=-M\left(V \Lambda^{2} W^{T}\right)+M\left(V \Lambda^{3} W^{T}\right) M$

with modal condition

$0=V W^{T}$

These relationships are used in the next section for solution of an model updating.

\section{$\mathbf{2 . 3}$ Inverse Formulas}

In this section, inverse formulas and conditions for given spectral and modal data to determine symmetric coefficient matrices are given in [8], [11]. Inverse formulas specify the coefficient matrices $\tilde{K}$ and $\tilde{D}$ in terms of the modal matrices $\boldsymbol{V}, \boldsymbol{W}$ and spectral one $\Lambda$. Using (13) and (21) we obtain $A=X \Lambda X^{-1}=\left[\begin{array}{c}V \\ V \Lambda\end{array}\right] \Lambda X^{-1}=\left[\begin{array}{c}V \Lambda X^{-1} \\ V \Lambda^{2} X^{-1}\end{array}\right]=$ $\left[\begin{array}{cc}0 & I \\ -\tilde{K} & -\tilde{D}\end{array}\right]$

By comparing the blocks in Eq. (28), it follows that

$\left[\begin{array}{ll}0 & I\end{array}\right]=V \Lambda X^{-1}$

and

$[-\tilde{K}-\tilde{D}]=V \Lambda^{2} X^{-1}$

After some manipulation the following inverse formulas are obtained

$\tilde{D}=-V \Lambda^{2} W^{T}$

$\tilde{K}=\tilde{D}^{2}-V \Lambda^{3} W^{T}$
$V \Lambda W^{T}=I$, and $V W^{T}=0$

Unfortunately there is no guarantee at this point that the formulas given by Eqs. (31) and (32) will result in $\tilde{D}$ and $\tilde{K}$ being symmetric.

The formulas (31) and (32) determine the two desired coefficient matrices of the system (2) if the third is chosen with spectral and modal properties which must satisfy the conditions (29) or (33). From condition (33), suitable matrices $\Lambda, \boldsymbol{V}$ and $\boldsymbol{W}$ can be specified to solve the inverse eigenvalue problem.

If the spectral and modal properties are chosen such that Eqs. (29) or (33) is valid, then the system determined by the coefficient matrices $\tilde{D}$ and $\tilde{K}$ is unique. Eqs. (31) and (32) solve the inverse problem for system with rigid body modes, i.e., $\tilde{D}$ and $\tilde{K}$ can be singular; however, these equations do not guarantee that the matrix coefficients are symmetric.

\subsection{Inverse Formulas for Symmetric Coefficient Matrices}

If the inverse formulas are to generate real symmetric coefficient matrices, then the specified spectral and modal matrices must fulfill some further requirements. From the theory of matrix polynomials [3] it is known that if a monic matrix polynomial is self-adjoint, then there exist a Jordan triple $\left(\boldsymbol{U}, \Lambda, \boldsymbol{P}_{\Lambda}, \boldsymbol{U}^{*}\right)$, where $\boldsymbol{P}_{\Lambda}$ is a permutation matrix and $\boldsymbol{U}^{*}$ denotes the complex conjugate transpose of the matrix $\boldsymbol{U}$. Hence in the case of interest here the spectral matrix $\Lambda$ and modal matrices $\boldsymbol{V}$ and $\boldsymbol{W}$ will generate hermitian coefficient matrices if the left modal matrix is of the form

$W^{T}=P \Lambda V^{*}$

where the real valued matrix $\boldsymbol{P}_{\Lambda}$ is given in [3].

The conditions that must be satisfied by the specified spectral and modal matrices in order to generate real symmetric coefficient matrices are presented next. From Eq. (15)

$X W^{T}=\left[\begin{array}{l}0 \\ I\end{array}\right]$

Substituting $X=\left[\begin{array}{c}V \\ V \Lambda\end{array}\right]$ and $\boldsymbol{W}^{\mathrm{T}}$ from (34) yields that

$\left[\begin{array}{c}V \\ V \Lambda\end{array}\right] P \Lambda V^{*}=\left[\begin{array}{l}0 \\ I\end{array}\right]$

with the conditions 
The conditions given by Eqs. (33) specify vectors that will generate real symmetric coefficient matrices for the given spectral matrix $\Lambda$. Note that this also depends on the structure of the state matrix, whether or not $\boldsymbol{A}$ is assumed to be simple or of general Jordan structure, and whether or not the dynamic system is over-damped, under damped, critically damped, or exhibits mixed damping.

$\boldsymbol{n} \times \boldsymbol{n}$ matrix $\boldsymbol{C}$ is real valued and nonsingular. This represents a choice and offers potential for restricting the solution of the inverse problem. Substituting this value for $\boldsymbol{V}_{\mathbf{i}}$ into Eq. (33) yields the simplified form [8]

$$
\begin{aligned}
& 2 V_{r} E V_{r}^{T}=-V_{R} P_{R} V_{R}^{T} \\
& 2 V_{r} F V_{r}^{T}=I-V_{R} J_{R} P_{R} V_{R}^{T}
\end{aligned}
$$

where $\boldsymbol{I}$ is the identity matrix and the matrices $\boldsymbol{E}$ and $\boldsymbol{F}$ are defined by

$$
\begin{aligned}
& E=P_{C}-C P_{C} C^{T} \\
& F=J_{r} P_{C}-C J_{i} P_{C}-J_{i} P_{C} C^{T}-C J_{r} P_{C} C^{T}
\end{aligned}
$$

The above formulas can now be used to construct a symmetric solution to the inverse eigenvalue problem as developed in the following section.

\section{Application of the Inverse Problem to the Model Updating}

Several incompatibilities exist between analytical models and experimentally obtained data. For instance consider the case of finite element analysis (FEA) modeling compared with experimental modal analysis (EMA) data [6]. This case accounts for the majority of activity in vibration modeling used in industry. In this situation the analytical model is characterized by a large number of degrees of freedom (and hence modes), ad hoc damping mechanisms and real eigenvectors (mode shapes).

The FEM model produces a mass, damping and stiffness matrix which is numerically solved for modal data consisting of natural frequencies, mode shapes and damping ratios. Common practice in industry is to compare this analytically generated modal data with natural frequencies, mode shapes and damping ratios obtained from EMA [6]. The EMA data is characterized by a small number of modes, incomplete and complex mode shapes and non proportional damping.

It is very common in practice for this experimentally obtained modal data to be in disagreement with the analytically derived modal data. The point of view taken is that the analytical model is in error and must be refined or corrected based on experimental data.

The goal of this chapter proposed here recognizes that the model correction problem is a subset of the inverse problem. The approach proposed here is to use the results of inverse eigenvalue problems to develop methods for correcting models.

The relation among the matrix coefficients $\boldsymbol{M}$, $\boldsymbol{D}$, and $\boldsymbol{K}$ and design parameters and the element matrices can be expressed follows

$M=\sum_{j=1}^{N m} m_{j} M_{e j}, D=\sum_{j=1}^{N_{d}} d_{j} D_{e j}, K=\sum_{j=1}^{N k} k_{j} K_{e j}$ (41)

where $\mathbf{k}_{\mathrm{i}^{\prime}} \mathbf{d}_{\mathrm{i}^{\prime}} \mathbf{m}_{\mathrm{i}}$ are the design parameters numbering $\mathbf{N}_{\mathbf{k}^{\prime}}, \mathbf{N}_{\mathbf{d}^{\prime}} \mathbf{N}_{\mathbf{m}}$ and $\mathbf{K}_{\mathbf{e j}} \mathbf{D}_{\mathbf{e j}} \mathbf{M}_{\mathbf{d j}}$ respectively and the element matrices are known.

\subsection{Systems with Proportional Damping}

For the case of symmetric coefficient matrices $\boldsymbol{K}, \boldsymbol{D}$, $\boldsymbol{M}$ and systems of simple structure $\left(\Lambda=S=\Lambda^{\mathrm{T}}\right)$ it follows from the eigenvalue problem that $\boldsymbol{W}=\boldsymbol{V}$. In the case of proportional damping where the damping matrix is of the form

$D=\alpha M+\beta K$

there exists such real matrix $\boldsymbol{V}_{\mathrm{O}^{\prime}}$, that the following hold

$V_{O}^{T} K V_{O}=\Omega_{o}^{2}$,

$V_{O}^{T} D V_{O}=2 \Delta$,

$V_{O}^{T} M V_{O}=I$,

\subsection{Complete Modal and Spectral Data Case}

For known modal and spectral data the matrix coefficients are given by Eqs.(24), (25) and (26). Then by using relations in Eq.(42) for known element matrices $\boldsymbol{M}_{\mathrm{e}^{\prime}} \boldsymbol{D}_{\mathrm{e}}$ and $\boldsymbol{K}_{\mathrm{e}}$ we yield the design parameters $\boldsymbol{m}_{\mathrm{i}^{\prime}} \boldsymbol{d}_{\mathrm{j}}$ and $\boldsymbol{k}_{\mathrm{j}}$ of the system. Entries in the matrices $\boldsymbol{M}, \boldsymbol{D}$ and $\boldsymbol{K}$ are given by linear combination of the design parameters as follows

$$
\begin{aligned}
& A_{K I}\left\{k_{l}\right\}=b_{K I}, A_{D I}\left\{d_{l}\right\}= \\
& b_{D I}, A_{M I}\left\{m_{l}\right\}=b_{M I}
\end{aligned}
$$

where $A_{\mathrm{KI}}, A_{\mathrm{DI}}, A_{\mathrm{MI}}$ to be $\left(N^{2}, N_{\mathrm{K}}\right),\left(N^{2}, N_{\mathrm{d}}\right)$ and 
$\left(N^{2}, N_{\mathrm{m}}\right)$ matrices and $\boldsymbol{b}_{\mathrm{KI}^{\prime}} \boldsymbol{b}_{\mathrm{DI}^{\prime}} \boldsymbol{b}_{\mathrm{MI}}$ to be $\left(N^{2}, I\right)$ vectors.

Let us have the analytical model given by the coefficient matrices $\boldsymbol{M}_{\mathrm{a}^{\prime}} \boldsymbol{D}_{\mathrm{a}}$ and $\boldsymbol{K}_{\mathrm{a}}$ with in advanced structure ( some entries are zeros ). For this model the modal and spectral data are represented by the matrices $\boldsymbol{V}_{\mathrm{a}^{\prime}} \mathbf{W}_{\mathrm{a}}$ and $\Lambda_{\mathrm{a}}$.

Let the experimental spectral and modal data be given by the matrices $\boldsymbol{V}_{\mathrm{e}^{\prime}} \boldsymbol{W}_{\mathrm{e}}$ and $\Lambda_{\mathrm{e}}$. By using Eqs. (24)-(26) we yield the experimental coefficient matrices $\boldsymbol{M}_{\mathrm{e}^{\prime}} \boldsymbol{D}_{\mathrm{e}}$ and $\boldsymbol{K}_{\mathrm{e}}$ which will have different structure than the analytical form of these matrices.

$K_{a}=\left[\begin{array}{ccccc}k_{1}+k_{2} & -k_{2} & 0 & 0 & 0 \\ -k_{2} & k_{2}+k_{3}+k_{6} & -k_{3} & 0 & -k_{6} \\ 0 & -k_{3} & k_{3}+k_{4} & -k_{4} & 0 \\ 0 & 0 & -k_{4} & k_{4}+k_{5} & 0 \\ 0 & -k_{6} & 0 & 0 & k_{6}\end{array}\right]$

where $^{k_{a j}}=1000(1.6,30,1.2,25,1.5,1.0)$

$$
m_{a j}=(0.5,5.5,5.5,0.8,3.0)
$$

Let us simulate errors in some design parameters and than build coefficient matrices $\boldsymbol{M}_{\mathbf{r}^{\prime}} \boldsymbol{D}_{\mathbf{r}}$ and $\boldsymbol{K}_{\mathbf{r}^{\prime}}$

where $k_{j r}=1000(1.84,25.5,1.056,29,1.68,1.14)$

$$
m_{r j}=(0.57,4.95,5.94,0.76,3.3)
$$

and $\alpha_{\mathbf{r}}=0.011, \beta_{\mathbf{r}}=0.0001$.

Then the design parameters determined from, and $\boldsymbol{K}_{\mathrm{e}}$ by using Eq.(41) will create coefficient matrices $\boldsymbol{M}_{\mathbf{u}^{\prime}} \boldsymbol{D}_{\mathbf{u}}$ and $\boldsymbol{K}_{\mathbf{u}}$ which will be different from both analytical $\boldsymbol{M}_{\mathrm{a}^{\prime}} \boldsymbol{D}_{\mathrm{a}}$ and $\boldsymbol{K}_{\mathrm{a}}$ and experimental $\boldsymbol{M}_{\mathrm{e}^{\prime}} \boldsymbol{D}_{\mathrm{e}}$ and $\boldsymbol{K}_{\mathrm{e}}$. The structures of the matrices $\boldsymbol{M}_{\mathbf{u}^{\prime}}$ $\boldsymbol{D}_{\mathrm{u}}$ and $\boldsymbol{K}_{\mathrm{u}}$ and matrices $\boldsymbol{M}_{\mathrm{a}^{\prime}} \boldsymbol{D}_{\mathrm{a}}$ and $\boldsymbol{K}_{\mathrm{a}}$ will be the same. Nonzero entries of matrices $\boldsymbol{M}_{\mathbf{u}^{\prime}} \boldsymbol{D}_{\mathbf{u}}$ and $\boldsymbol{K}_{\mathbf{u}}$ and nonzero entries of matrices $\boldsymbol{M}_{\mathrm{e}^{\prime}} \boldsymbol{D}_{\mathrm{e}}$ and $\boldsymbol{K}_{\mathrm{e}}$ will be the same $\left(\boldsymbol{N}_{\mathrm{k}^{\prime}} \boldsymbol{N}_{\mathrm{d}^{\prime}}, \boldsymbol{N}_{\mathrm{m}}=\boldsymbol{n}_{\mathrm{bK}^{\prime}} \boldsymbol{n}_{\mathrm{bD}^{\prime}} \boldsymbol{n}_{\mathrm{bM}^{\prime}}\right)$, or will be different in the sense of minimum of Euclidian norm $\left(\boldsymbol{N}_{\mathrm{k}^{\prime}} \boldsymbol{N}_{\mathrm{d}^{\prime}} \boldsymbol{N}_{\mathrm{m}}<\boldsymbol{n}_{\mathrm{bK}^{\prime}} \boldsymbol{n}_{\mathrm{bD}^{\prime}}, \boldsymbol{n}_{\mathrm{bM}}\right)$. Because the structure of the matrices $\boldsymbol{M}_{\mathbf{u}^{\prime}} \boldsymbol{D}_{\mathbf{u}}$ and $\boldsymbol{K}_{\mathbf{u}}$ and the matrices $\boldsymbol{M}_{\mathrm{e}^{\prime}} \boldsymbol{D}_{\mathbf{e}}$ and $\boldsymbol{K}_{\mathbf{e}}$ is different, the matrices $\boldsymbol{V}_{\mathbf{u}^{\prime}} \boldsymbol{W}_{\mathbf{u}}$ and $\Lambda_{\mathbf{u}}$ and matrices $\boldsymbol{V}_{\mathrm{e}^{\prime}} \boldsymbol{W}_{\mathrm{e}}$ and $\Lambda_{\mathrm{e}}$ will be different too.

\subsection{Example}

Let the coefficient matrices of the analytical system are

$$
\begin{aligned}
& M_{a}=\operatorname{diag}\left(m_{1}, m_{2}, m_{3}, m_{4}, m_{5}\right) \\
& D_{a}=\alpha M+\beta K
\end{aligned}
$$

where

$\alpha_{a}=0.01, \beta_{a}=0.0001$

This system will by represented by modal and spectral matrices $\boldsymbol{V}_{\mathbf{r}^{\prime}} \boldsymbol{W}_{\mathbf{r}}$ and $\Lambda_{\mathbf{r}}$ or $\boldsymbol{V}_{\mathbf{0 r}}$ and $\boldsymbol{S}_{\mathbf{r}^{\prime}}$. By using Eqs.(24), (25), and (26), or (43)-(45) we compute the effective design parameters with errors.

Let us define some errors matrices $\boldsymbol{e} \boldsymbol{V}_{0}=\boldsymbol{e} \boldsymbol{W}_{0} . \boldsymbol{e S}$ representing a noise in measurement and ones.

These will represent experimental modal and spectral data $\boldsymbol{V}_{0 \mathrm{e}}=\boldsymbol{V}_{0 \mathrm{r}} \cdot \boldsymbol{e} \boldsymbol{V}_{0}$ and $\boldsymbol{S}_{\mathrm{e}}=\boldsymbol{S}_{\mathrm{r}} \cdot \boldsymbol{e} \boldsymbol{S}$. Multiply every entry of the error matrices with corresponding entry of effective modal and spectral ones.

By using Eqs.(43)-(45) we yield the experimental coefficient matrices $\boldsymbol{K}_{\mathrm{e}^{\prime}} \boldsymbol{M}_{\mathrm{e}}$ and $\alpha_{\mathrm{e}}=0.009$, $\beta_{\mathrm{e}}=0.0001$.

From (46) we yield the new design parameters (see [8])

$$
\begin{aligned}
& k_{u j}=10^{4}[0.26,2.36,0.13,3.07,0.09,0.09] \\
& m_{u j}=[0.53,4.80,6.27,0.77,3.52] \\
& \alpha_{a}=0.01, \beta_{a}=0.0001, \alpha_{r}=0.011, \\
& \beta_{r}=0.0001, \alpha_{e}=0.009, \beta_{e}=0.0001
\end{aligned}
$$

From the Table 1 follows, that compare to effec-

\begin{tabular}{|c|c|c|c|c|c|c|}
\hline$k_{\mathrm{aj}}$ & 1.6 & 30 & 1.2 & 25 & 1.5 & 1.0 \\
\hline$k_{i j}$ & 1.84 & 25.5 & 1.056 & 29 & 1.68 & 1.14 \\
\hline$k_{u j}$ & 2.686 & 23.665 & 1.397 & 30.793 & 0.951 & 0.959 \\
\hline $\mathrm{m}_{\mathrm{aj}}$ & \multicolumn{2}{|l|}{0.5} & 5.5 & \multicolumn{2}{|c|}{0.8} & 3.0 \\
\hline$m_{i j}$ & 0.57 & \multicolumn{2}{|c|}{4.95} & \multicolumn{2}{|c|}{0.76} & 3.3 \\
\hline$m_{\mathrm{uj}}$ & 0.5308 & \multicolumn{2}{|c|}{4.8068} & & 737 & 3.5202 \\
\hline
\end{tabular}
tive design parameters updated are under some errors which are relative small for mass design parameters (about 5-7\%) and little high for stiffness ones (up to 45\%). It should be notice, that for complete spectral and modal data case without noise the inverse formulas give exact design parameters.

Table 1: Comparison of analytical, effective and updated design parameters. 


\section{Conclusion}

This paper summarize the authors previous effort on inverse eigenvalue problem.

The conditions (27) and (33) for given spectral and modal data have been defined under which the inverse formulas determine real symmetric coefficient matrices $\tilde{D}$ and $\tilde{K}$. However, that solution does not preserve given eigenvectors. The alternative solution extends these results to include the preservation of eigenvectors. There have been derived conditions under which spectral and modal data determine real, symmetric coefficient matrices $\tilde{D}$ and $\tilde{K}$ which do not necessarily commute. The method has been outlined which allows the synthesis of a symmetric, underdamped linear system having desired eigenvalues and eigenvectors.

The new result gives the alternative solution to the inverse eigenvalue problem in vibration in $\mathbf{2 n}$ space to include the determination of all three coefficient matrices $\boldsymbol{M}, \boldsymbol{D}$ and $\boldsymbol{K}$ from given spectral and modal data and to apply the inverse eigenvalue problem to the model updating problem.

Updating methods for the cases of complete spectral and modal data, incomplete spectral and modal data with complete eigenvectors and incomplete modal and spectral data with incomplete eigenvectors are presented.

There are three main objectives of the next research:

- the reconstruction of mass, damping and/or stiffness matrices using incomplete modal data

- developing inverse procedures which retain the connectivity of the physical mode

- investigation of the sensitivity on the resulting inverse methods to the quality and quantity of measured vibration data.

\section{Acknowledgement}

The first author gratefully acknowledges the support of the Slovak Ministry of Education Grant Agency VEGA \# 1/0744/10.

\section{References}

[1] Danek, O. (1982). Non conservative Dynamic Systems. Strojnícky Časopis, vol. 33, no. 6, pp. 667-680

[2] Starek, L., Inman, D.J. (2004). Design of Nonproportional Damped Systems via Symmetric Inverse Problem. Journal of Vibration and Acoustic, vol. 126, pp. 212-219

[3] Gohberg, I., et al. (1982). Matrix Polynomials. Academic Press

[4] Gladwell, G.M.L. (1986). Inverse Problem in Vibration. Marti- nus Nijhoff Publishers, Boston, MA

[5] Lancaster, P., Maroulas, J. (1987). Inverse Eigenvalue Problems for Damped Vibrating Systems.Journal of Mathematical Analysis and Application, vol.123, no.1, pp. 238-261

[6] Lieven, N.A.J., Ewins, D.J. (1990). Expansion of Modal Data for Correlation. 8th IMAC, pp. 605-609

[7] Starek, L., Inman, D.J. (1991). On the Inverse Vibration Problem with Rigid Body Modes. ASME Journal of Applied Mechanics, vol.58, pp. 1101-1104

[8] Starek, L., Inman, D.J., Kress A. (1992). A Symmetric Inverse Vibration Problem. ASME Journal of Vibration and Acoustics, vol.114, pp. 564-568

[9] Starek L., Inman D.J. (1995). A Symmetric Inverse Vibration Problem with Over-damped modes. Journal of Sound and Vibration, vol. 181, no. 5, pp. 893-903

[10] Starek, L., Inman, D.J., Pilkey, D.J. (1995). A Symmetric Positive Definite Inverse Vibration Problem with Underdamped Modes. Proc. of Design Engineering Technical Conference, Boston, Messachusetts, DE, vol. 84-3, volume 3-Part C, ASME, pp. 1089-1094

[11] Starek, L., Inman, D.J. (1997). A Symmetric Inverse Vibration Problem for Non-proportional, Underdamped System. ASME Journal of Applied Mechanics, vol. 64, no. 3, pp. 601605 


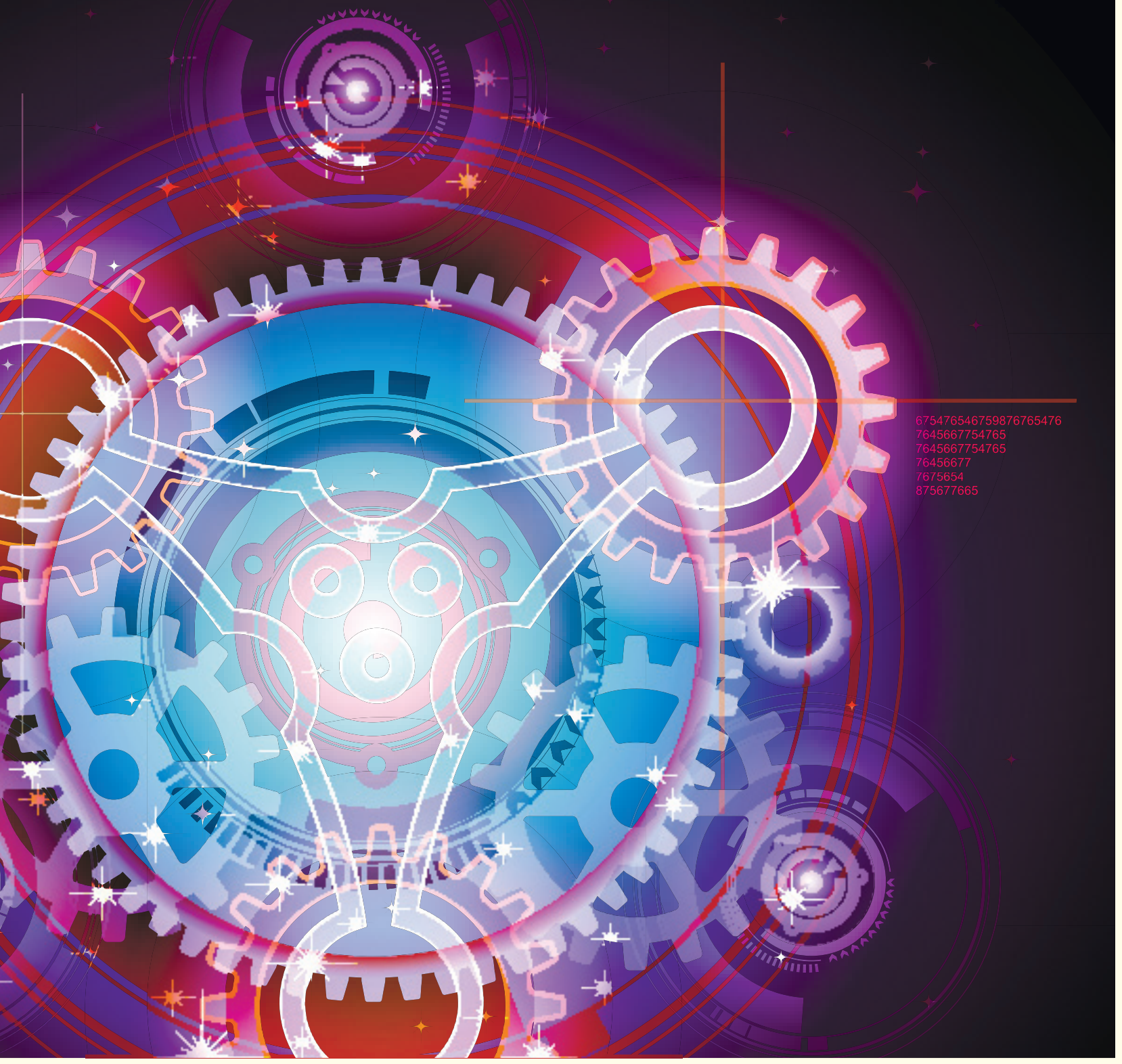

